\section{Some Adaptive Control Problems Which Convert to a "Classical" Problem in Several Complex Variables}

\author{
J. William Helton
}

\begin{abstract}
In this note, we discuss the equivalence of what we refer to as bi- $H^{\infty}$ control problems to certain problems of approximation and interpolation by analytic functions in several complex variables. We now introduce what we mean by bi- $H^{\infty}$ control. The goal is to perform an $H^{\infty}$ control design for a plant where part of it is known and a stable subsystem $\delta$ is not known, that is, the response of the plant at "frequency" $s$ is $P(s, \delta(s))$. We assume that once our control (closed loop) system is running, we can identify the subsystem $\delta$ on line. Thus the problem is to design a function $K$ offline that uses this information to produce a $H^{\infty}$ controller via the formula $K(s, \delta(s))$. The challenge is to pick $K$ so that the controller yields a closed loop system with $H^{\infty}$ gain at most $\gamma$ no matter which $\delta$ occurs. This is entirely a frequency domain problem, which has the flavor of some types of gain scheduling or control which adapts to slow variations. The bulk of this article is devoted to showing how several bi- $H^{\infty}$ control problems convert to two complex variable interpolation problems. These precisely generalize the classical (one complex variable) interpolation (AAK-commutant lifting) problems which lay at the core of $\boldsymbol{H}^{\infty}$ control. These problems are hard, but the last decade has seen substantial success on them in the operator theory community. In the most ideal of bi- $H^{\infty}$ cases these lead to a necessary and sufficient treatment of the control problem.
\end{abstract}

Index Terms-Adaptive $H^{\infty}$, gain scheduling in frequency domain, several complex variables.

\section{INTRODUCTION}

The key mathematical optimization problem which underlies the beginnings of $H^{\infty}$ control was solved by mathematicians working on the complex variables side of operator theory, cf. [9]. They produced extremely powerful and useful Nevannlina-Pick, analytic function approximation, interpolation and commutant lifting formulas. $H^{\infty}$ control theory soon had a life of its own and while the mathematicians in operator theory participated for about a decade, a large body of them and their students ultimately moved into several complex variables (SCVs), generalizations of Nevanlinna-Pick and related problems which were completely removed from systems engineering. Much of this effort in SCV focuses on approximation with functions analytic in several complex variables. This is an extremely hard subject and the results are not nearly so satisfying as in the case of one complex variable. However, there is some impressive progress and fine theorems.

This article presents an idea for applying some of these approximation results in SCV to bi- $H^{\infty}$ control problems. The hope is that specialists in the two rather widely separated topics arising here will pursue this area further. The author is a specialist in neither area.

\section{The MATHEMATICAL IngREdiEnTS}

\section{A. Interpolation in $\mathrm{SCV}$}

Of particular interest is a theorem due to J. Agler [1] and extensions of it [3], [4]. Let Disk ${ }_{n}$ denote the unit polydisk $\left\{\left(v_{1}, \ldots, v_{n}\right):\left\|v_{1}\right\| \leq 1, \ldots,\left\|v_{n}\right\| \leq 1\right\}$ in $\mathbf{C}^{n}$ the space of $n$ complex variables. Let $B H^{\infty}(n)$ denote the functions analytic on

Manuscript received September 21, 1998; revised April 7, 2000 and February 25, 2001. Recommended by Associate Editor J. Chen. This work was supported in part by the National Science Foundation, the ONR, the Ford Motor Company, and the CRDF.

The author is with the University of California at San Diego, La Jolla, CA 92093 USA.

Publisher Item Identifier S 0018-9286(01)11083-4.
$D_{i s k_{n}}$ and bounded in supremum norm by 1 . Also $M_{k \times \ell}$ denotes the $k \times \ell$ matrices, $B M_{k \times \ell}$ denotes the unit ball in $M_{k \times \ell}$. The Nevannlina-Pick type interpolation problem on $D i s k_{2}$, the bi-disk, is:

Given a finite set of points $\left\{\left(s_{k}, w_{k}\right)\right\}$ in the bi-disk and numbers $z_{k}$ in the complex plane $\mathbf{C}$, and a positive number $\gamma$, find a function $f$ in $\gamma B H^{\infty}(2)$ which takes the values $z_{k}$ at the points $\left\{\left(s_{k}, w_{k}\right)\right\}$.

Theorem 2.1: The Nevannlina-Pick interpolation problem on the bi-disk has a solution $f$ in $\gamma B H^{\infty}$ (2) if and only if the linear matrix inequality (LMI)

$\left(\gamma^{2}-\bar{z}_{k} z_{\ell}\right)=\left(1-\bar{s}_{k} s_{\ell}\right) \Lambda_{k \ell}+\left(1-\bar{w}_{k} w_{\ell}\right) \Omega_{k \ell}$

$\Lambda$ and $\Omega$ positive semidefinite matrices

has a solution. The solution is a rational function $f(\cdot, \cdot)$ in both variables $s, w$.

We point out a second form of the Agler inequality (1) which has fewer unknowns and so is probably more efficient for LMI software solution. This is:

Find $\Omega$ positive semidefinite, so that the selfadjoint matrix with $k, \ell$ th entry

$$
\frac{\left(\gamma^{2}-\bar{z}_{k} z_{\ell}\right)}{\left(1-\bar{s}_{k} s_{\ell}\right)}-\frac{\left(1-\bar{w}_{k} w_{\ell}\right)}{\left(1-\bar{s}_{k} s_{\ell}\right)} \Omega_{k \ell}
$$

is positive semidefinite. A condensed notation for this is

$$
0 \leq S-P \circ_{s} \Omega
$$

where $o_{s}$ denotes the Schur product (entry by entry) product of two matrices.

The proof of Agler's Theorem is in principle constructive, see [3] and [4], though considerable effort would probably be required to refine it for computer implementation. While Theorem 2.1 is only stated for $f$ a scalar valued function, it holds in a very similar form when $f$ is a matrix valued function, see [4]. However, this article involves only SISO systems, primarily for ease of pedagogy.

To derive the second form of the Agler inequality (2) from (1), just divide (1) by $\left(1-\bar{s}_{k} s_{\ell}\right)$ and solve for $\Lambda_{k, \ell}$ to obtain that it equals (2).

\section{B. Bi-Disk Model Matching Problem}

Such interpolation problems arise naturally when one is trying to find a function $f$ analytic on the bi-disk which has a supremum norm $\leq \gamma$ and which has the form

$$
f=A+B^{1} h_{1}+B^{2} h_{2}
$$

for some $h_{1}, h_{2}$ analytic on the bi-disk. This is because in the typical situation on the bi-disk the zero set $Z_{g}:=\{(v, w): g(v, w)=0\}$ of a function $g$ in $B H^{\infty}(2)$ is empty or is the union of finitely many one complex dimensional surfaces. Thus, $B^{1}$ and $B^{2}$ have zero sets intersecting (transversally) in only a finite number of points $\left\{s_{k}, w_{k}\right\}$ and at these points $f$ is constrained to take values

$$
f\left(s_{k}, w_{k}\right)=z_{k}:=A\left(s_{k}, w_{k}\right) \quad \forall k=1, \ldots v
$$

regardless of what $h_{1}, h_{2}$ are. An expert in $H^{\infty}$ control will recall that this problem on the ordinary disk (as opposed to the bi-disk) will have $B^{2}=0$ and is the model matching problem. Consequently, we shall call problem (4) the bi-disk model matching problem.

A symmetry which occurs in engineering functions, which we shall call bi-real, is

$$
g: \operatorname{Disk}_{2} \rightarrow \mathbf{C} \quad \text { satisfies } \quad g(\bar{s}, \bar{w})=\overline{g(s, w)} \quad \forall s, w \in \mathbf{C} .
$$




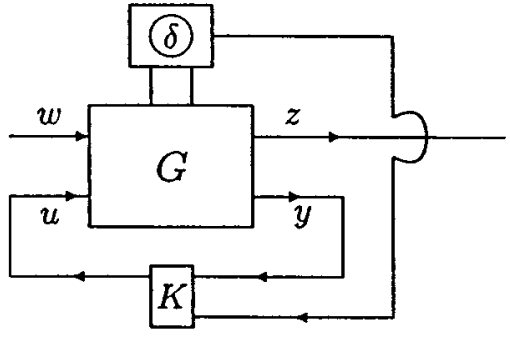

Fig. 1. $\mathrm{Bi}-H^{\infty}$ controller.

Corollary 2.2: Given functions $A, B^{1}, B^{2}$ analytic on the bi-disk having $B^{1}, B^{2}$ whose zero sets intersect transversally in a finite number of points $\left\{\left(s_{k}, w_{k}\right)\right\}$ and meet a technical (generically true general position) hypothesis on how they cross the boundary of $D_{i s k_{2}}$. Let $z_{k}:=A\left(s_{k}, w_{k}\right)$. The bi-disk model matching problem has a solution $f$ in $B H^{\infty}(2)$ (and bi-rational) if and only if the Linear Matrix Inequality (2) has a solution. If $A, B^{1}, B^{2}$ are bi-real, then we may take $f$ to be bi-real.

Proof: This is an immediate consequence of Agler's Theorem up to the part involving the bi-real property. If $A, B^{1}, B^{2}$ are bi-real, then the set $\mathcal{I}:=\left\{\left(s_{k}, w_{k}, z_{k}\right):\right.$ all $\left.k\right\}$ of interpolating data is unchanged by applying the conjugation symmetry

$$
(s, w, z) \rightarrow(\bar{s}, \bar{w}, \bar{z}) .
$$

Thus if $f \in B H^{\infty}(z)$ satisfies the interpolation constraints given by $\mathcal{I}$, then $\tilde{f}(s, w):=(1 / 2)[\overline{f(\bar{s}, \bar{w})}+f(s, w)]$ does also and solves the bi-disk interpolation problem for the same $\mathcal{I}$.

\section{The Bi-VARiable Control Problem}

Consider the rather standard picture of a controlled discrete time system with an uncertainty $\delta$, (see Fig. 1). In our approach, the uncertainty is presented in terms of its discrete time frequency response function (FRF), denoted $\delta(s)$.

We shall take $\delta$ to be an uncertainty which is a stable SISO system whose supremum norm over the unit disk is less than or equal to $d_{0}$. We wish to design a controller $K$ offline and the ground rules presume that once the system is running the controller can identify $\delta$, that is, we take $K$ to have the form

$$
K(s, \delta(s))
$$

We call this a bi-variable controller. We shall always take $w$ and $z$ to be 1 dimensional signals and $\delta$ to be SISO, but the controller $K$ can take values which are matrices of any dimension. We shall take the convention that the FRF of a stable system is analytic inside the disk, in order to be consistent with the mathematical part.

Our approach, under the assumption

$$
M A N Y \delta s\{(\mathrm{~s}, \delta(\mathrm{s})): \text { all } \delta \text { which arise and all }|\mathrm{s}| \leq 1\}
$$

in principle gives a necessary and sufficient theory for control design. In practice there often will be compromises in getting particular problems to meet the (MANY $\delta \mathrm{s}$ ) condition, but these compromises occur later in the design rather than at the core of the theory, as happens with other approaches to adapting to plant variations. This may offer some advantages. For example, if (MANY $\delta$ s) fails, then a graphical display (frequency by frequency) of the set above would tell how much it fails and at what frequencies. This might be informative.

It is a simple variation of our theory to allow $d_{0}$ to be a smooth nonnegative function of $s$ (which has a spectral factorization); this allows a little flexibility. More elaborate variations might involve rescaling by

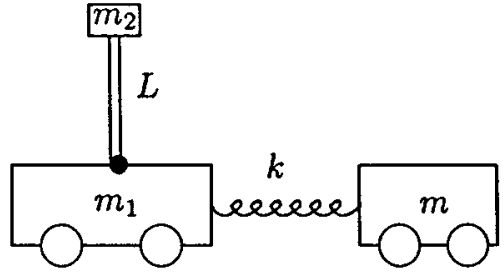

Fig. 2. Two carts.

a class of frequency dependent conformal maps (tricky business), cf. T. Sideris's unpublished thesis or [14].

\section{A. Physical Set Up}

We mention a few physical situations where the bi- $H^{\infty}$ setup might arise. Our discussion has the tone of gain scheduling or adaptive control which adapts to a slowly varying subsystem or environment. More detailed studies and richer classes of examples are best left to engineers.

Suppose we build a machine $G$ and wish to sell it to many different customers who will be docking or loading $G$ with many different loads $\delta$. When we sell $G$ we must also provide a controller along with it which works for a broad range of loads. The customer before he attaches a load $\delta$ to $G$ must determine its response function $\delta$ by modeling or with a spectrum analyzer. For such situations a bi-variable controller $K(\cdot, \cdot)$ is a reasonable type of controller for our company to provide along with $G$.

Many variations on implementation are possible and we sketch one now primarily for tutorial reasons. Once the customer determines $\delta$, either by finding model parameters $\delta_{i}$ or as spectrum analyzer data, the (discrete time) function $\delta$ could be stored in many ways, for example, as data $\left\{e^{i \theta_{k}}, \delta\left(e^{i \theta_{k}}\right)\right\}$ on a grid on $[0,2 \pi]$. Our company's controller algorithm is software which contains a function $K(s, w)$, say stored as a two variable rational function. The controller $C$ which is implemented for $\delta$ loaded onto $G$ has frequency response function on the $\left\{e^{i \theta_{k}}\right\}$ grid equal to

$$
C\left(e^{i \theta_{k}}\right)=K\left(e^{i \theta_{k}}, \delta\left(e^{i \theta_{k}}\right)\right)
$$

which can be numerically discrete Laplace transformed to produce the impulse response $c(t)$. The controller can be implemented by convolving $c$ with the measurement $y$.

Moreover, if loads are passive, they meet the assumption $\delta \in B H^{\infty}(1)$, and if assumption (MANY $\delta \mathrm{s}$ ) below holds, the theory is in principle necessary and sufficient. A situation which gives some conceptual perspective is that of a turning mill; the turning mill (together with weights the designer chooses to achieve performance) corresponds to $G$. Work of different masses and elasticities is placed on the turning mill. The load is passive, so its FRF lies in $B H^{\infty}(1)$.

There is considerable freedom in how we use $\delta$ in Fig. 1. We could have a plant which is linear and completely known except for a SISO subsystem $g$ which is uncertain at the time of the controller design. If all uncertain subsystems $g$ which occur will be close to a nominal $g_{0}$ in the sense

$$
\left|g\left(e^{i \theta}\right)-g_{0}\left(e^{i \theta}\right)\right| \leq d_{0}\left(e^{i \theta}\right) \quad \text { for all } \theta
$$

for a predetermined function $d_{0}$, then take $\delta:=g-g_{0}$ to put this situation into the form of Fig. 1. Bi-variable controllers seem reasonable for this type of control.

\section{B. A Tale of Two Carts}

To fix a more concrete image in the reader's mind consider two carts joined by a spring, see Fig. 2 . 
Cart ${ }_{1}$ is activated by torques on the wheels (this is the control input $u$ to the system), and it supports an inverted pendulum of length $L$ which we wish to keep vertical. Let us say we build cart $_{1}$, so everything about it is known; $L, m_{1}, m_{2}$, etc. Now cart 2 is made by someone else, and the controller we sell with cart 1 must be prepared to handle many spring sizes, masses as well as dampings. Our assumption about the cart $2 \mathrm{~s}$ which can occur is that there is a nominal one, with FRF equal to $g_{0}$, and that in the coordinates we are using each possible cart 2 has FRF $g$ whose distance from $g_{0}$ is less than some function $d_{0}$, that is

$$
\left|g_{0}\left(e^{i \theta}\right)-g\left(e^{i \theta}\right)\right| \leq d_{0}\left(e^{i \theta}\right)<\infty \quad s \in \text { Disk. }
$$

Note that since all carts are stable $\delta:=g_{0}-\tilde{g}$ is stable. This set up fits the mold of Fig. 1 with $\delta$ as above and with $G$ denoting the linearization of cart ${ }_{1}$ connected with nominal cart ${ }_{2}$, together with a port for the additive uncertainty $\delta$, and together with weights emphasizing performance the designer desires.

The key sets for testing the (MANY $\delta \mathrm{s}$ ) condition are

$$
\Delta_{d_{0}}:=\left\{(s, w):|s| \leq 1,|w| \leq\left|\tilde{d}_{0}(s)\right|\right\} \subset \mathbf{C}
$$

and

$$
\begin{aligned}
V_{\text {art }_{2}}:=\{( & \left(s, \delta_{\text {art }_{2}}(s)\right):|s| \leq 1 \\
& \left.\operatorname{cart}_{2} \subset \text { Admissible }\right\} \subset \Delta_{d_{0}} .
\end{aligned}
$$

Here $\tilde{d}_{0}$ denotes the minimum phase spectral factor of $d_{0}^{2}$. The fact $V_{\text {cart }_{2}} \subset \Delta_{d_{0}}$ follows from (7) and cart 2 being stable, and this means that the problem fits in our bi- $H^{\infty}$ setup. On the other hand if the reverse condition

$$
V_{\text {cart }_{2}} \supset \text { Boundary } \Delta_{d_{0}}
$$

holds, the bi- $H^{\infty}$ methods of this note are (up to computational difficulties and some issues of approximation) necessary and sufficient.

When condition (8) fails our bi- $H^{\infty}$ approach becomes conservative, but it is easy, for each $s$ in the right half plane, to graph cross sections

$$
\left.V_{\text {cart }_{2}}\right|_{s} \text { v.s. }\left.\quad \Delta_{d_{0}}\right|_{s} .
$$

This compares the uncertain loads which actually arise to the uncertain loads (of possibly more general configuration) which our controller handles.

\section{Manufacturing Variability}

In manufacturing processes there is great variability. Thus a mathematical model of the ideal product is mared by parasitics which actually occur. Suppose for the moment we manufacture and control devices which ideally consist of two decoupled linear systems $G_{1}$ and $G_{2}$ which in practice contain a parasitic influence $\delta$ of subsystem 1 on subsystem 2 . Over the course of the devices commercial production $\delta$ may vary greatly. If after each device is built we can determine $\delta$, then this problem would be a reasonable candidate for bi- $H^{\infty}$ control. By contrast if subsystem 2 also influences subsystem 1 , then this adds a second uncertain system $\delta_{2}$, which if unrelated to $\delta$, leads to a tri-disk $H^{\infty}$ interpolation problem," namely, one based on the tri-disk $\left\{\left(s, w_{1}, w_{2}\right)\right\}$. This is not a fully solved problem. Note that one might treat $\delta_{2}, \delta$ differently. Namely, study constraints of the form $\left|\delta_{2}\right|^{2}+|\delta|^{2} \leq d_{0}^{2}$. This has the advantage of producing a solvable $H^{\infty}$ interpolation problem. Such extensions of bi- $H^{\infty}$ theory are indicated in Section VI.

\section{Converting Bi $H^{\infty}$ Control to Bi-Disk Model Matching AND INTERPOLATION}

This note focuses on the offline design problem of finding a suitable bi- $H^{\infty}$ controller $K(\cdot, \cdot)$. Henceforth, set the uncertainty size parameter $d_{0}$ equal to 1 . The standard bi- $H^{\infty}$ control problem is

Find a bi- $H^{\infty}$ controller

$$
K: \mathbf{C} \times \mathbf{C} \rightarrow M_{p q}
$$

so that for each choice of $\delta \in B H^{\infty}(1)$, the controller $c(s)=$ $K(s, \delta(s))$ makes the closed-loop system in Fig. 1

1) internally stable;

2) have gain less than or equal to $\gamma$.

\section{A. Stability}

The next lemma shows how one can routinely go from stability for each $\delta \in H^{\infty}(1)$ to "stability" on Disk 2 . Bounded analyticity on $D_{i s k_{2}}$ we often call bi-stability.

Lemma 4.1: If $f(s, \delta(s))$ is analytic and uniformly bounded for all $s \in$ Disk and all ${ }^{1} \delta \in B H^{\infty}(1)$, then $f(s, w)$ is analytic and all uniformly bounded on the bi-disk.

One consequence of this lemma is that stability of the closed loop system for each $\delta$ is equivalent to the two variable transfer function $T(\cdot, \cdot)$ lying in $H^{\infty}(2)$.

\section{B. $H^{\infty}$ Gain}

Another objective of control is to achieve desired performance. This means find $K$ in Fig. 1 which makes

$$
|T(s, \delta(s))| \leq \gamma \quad \text { for all } s \in D i s k_{1} \text { and } \delta \in B H^{\infty}(1) .
$$

This is equivalent to

$$
|T(s, w)| \leq \gamma \quad \text { for all }(s, w) \in \operatorname{Disk}_{2} .
$$

$\gamma$ is called the gain of the closed loop system.

\section{Internal Stability}

Recall that internal stability of the system in Fig. 1 means that when input in is added to the $u$ loop and input $i_{y}$ to the $y$ loop, the nine closed loop transfer functions from $w, i_{u}, i_{y}$ to $z, u, y$ are stable (see [8, Ch. 4.2, Lemma 1]). Bi- $H^{\infty}$ control requires that all of these be stable for all $\delta \in B H^{\infty}(1)$. Lemma 4.1 then gives that each of these are bi-stable. The conversion of this to interpolation conditions is involved, so we merely show what happens for stable plants (an easy situation). In Section $\mathrm{V}$ we describe bi- $H^{\infty}$ classical control for plants which may or may not be stable.

\section{Model Matching for Stable G}

Denote the transfer function $G$ of the plant by

$$
G:=\left(\begin{array}{ll}
A(s, \delta(s)) & B(s, \delta(s)) \\
C(s, \delta(s)) & D(s, \delta(s))
\end{array}\right) .
$$

Here the functions $A, B, C, D$ are rational in two variables and take values as follows:

$$
\begin{array}{ll}
A(s, w) \in \mathbf{C} & B(s, w) \in M_{1 p} \\
C(s, w) \in M_{q 1} & D(s, w) \in M_{q p}
\end{array}
$$

${ }^{1}$ When $f(\cdot, \cdot)$ is rational the set of $\delta$ s for which this must hold need not be the entirety of $B H^{\infty}(1)$, but merely be so big as to satisfy condition (MANY $\delta \mathrm{s}$ ). To prove this as well as the lemma use Hartog's Theorem (see [12]). 
and the controller satisfies $K(s, w) \in M_{p q}$. Now we assume that $G$ is bi-stable to give a simple illustration of the role of model matching.

Lemma 4.2: Suppose $G$ is in $H^{\infty}(2)$, that is, $G$ is bi-stable. The bi- $H^{\infty}$ controllers $K(\cdot, \cdot)$ making the closed loop system in Fig. 1 internally stable for all $\delta \in B H^{\infty}(1)$ correspond precisely to the closed loop transfer functions $T$ of general model matching form

$$
T=A+B Q C
$$

for some $M_{p q}$ valued function $Q$ with entries in $H^{\infty}(2)$.

Idea of Proof: For each fixed $\delta$ the closed loop transfer function in Fig. 1 is

$$
T=\mathcal{F}_{G}(K):=A+B K(1-D K)^{-1} C .
$$

This gives representation (10) with $Q$, often called the closed loop controller, taken to be

$$
Q=K(1-D K)^{-1} .
$$

For this we need stability of $Q$, which follows from internal stability, since $Q$ is the transfer function from $i_{y}$ to $u$. Conversely, given $Q \in$ $H^{\infty}(1)$ construct $K$ by $K=(1+Q D)^{-1} Q$. Because of bi-stability of $G$ we have internal stability of the closed loop system as is standard in $H^{\infty}$ control. Since these stability facts hold for all $\delta \in H^{\infty}(1)$. We have by Lemma 4.1 that they hold with bi-stability replacing stability.

\section{E. Converting Model Matching to Interpolation}

In the previous section we reduced control problems to bi-disk model matching. In this and in the next section we convert several model matching problems to interpolation, thereby showing how Agler's Theorem can be used to solve them. These examples hopefully illustrate our main idea. Here we shall always take $\gamma=1$.

1) Two Controls-One Measurement: When the plant has 1 measurement output but $m$ control inputs, we take $Q$ to have a single input and $m$ outputs

$$
Q=\left(\begin{array}{c}
Q_{1} \\
\vdots \\
Q_{m}
\end{array}\right)
$$

This special structure of the plant makes $T$ have the form $T=A+$ $B Q C$ which when written out in coordinates is

$$
T=A+B_{1} Q_{1} C+\cdots+B_{m} Q_{m} C .
$$

For the standard problem to have a solution it is necessary that $A, B_{1}, \ldots, B_{m}, C \in H^{\infty}(2)$.

When $m=2$ and when $C$ has no zeros on the bi-disk, this is precisely what was called a bi-disk model matching problem in Section II-B, so it fits the mold of Agler's Theorem, since having a finite number of interpolating constraints is the typical situation. Thus we can apply LMI (2) to it to obtain a test to determine the limitations of control for the model matching problem. Construction of the controller requires implementing Theorem 2.1 constructively, which was discussed in Section II-A.

2) One Measurement-One Control: At last we mention the traditional $H^{\infty}$ control problem. Here we have $T=A+B Q C$. Thus the interpolating condition becomes

$$
T=A \quad \text { on } Z_{B C}
$$

where $Z_{B C}:=\{(s, w): B(s, w) C(s, w)=0\}$. This typically is a one complex dimensional curve in $\mathbf{C}^{2}$ or a finite set of such curves in $\mathbf{C}^{2}$. Thus we do not have a finite set of interpolating constraints, a situation to be discussed in Section V-A.

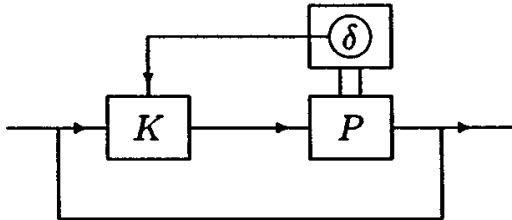

Fig. 3. Classical control via bi- $H^{\infty}$.

\section{Classical Control}

One can possibly follow the calculation in this section without having read Sections IV and IV-E. We treat a special case of the standard problem described by Fig. 3. Assume $P$ has poles and zeros of degree 1.

\section{A. The Method}

Here, we layout the procedure; later we give more details.

The closed-loop transfer function is $T(s, \delta(s))=P(s, \delta(s))$ $Q(s, \delta(s))$ where

$$
Q=K(1+P K)^{-1}
$$

and the controller has the form $K(s, \delta(s))$. The usual performance function is the mixed sensitivity function which has performance level $\tilde{\gamma}$ for all $\delta$ in $B H^{\infty}(1)$ if and only if

$$
\sup _{(s, w) \in D_{i s k_{2}}}\left[\left|\rho_{1}(s)(1-T(s, w))\right|^{2}+\left|\rho_{2}(s) T(s, w)\right|^{2}\right]
$$

is less than or equal to $\tilde{\gamma}^{2}$. Here $\rho_{1}, \rho_{2}$ are minimum phase (outer) weight functions, i.e., they have no poles or zeros in the disk. Some algebra (see Section V-A2 for formulas) and factoring convert this inequality to one of the form

$$
\begin{aligned}
& \mathcal{T}(s, w):=\alpha(s)+\beta(s) T(s, w) \quad \alpha, \beta \in H^{\infty}(1) \\
&|\mathcal{T}(s, w)| \leq \gamma \quad \text { all }(s, w) \in \operatorname{Disk}_{2}
\end{aligned}
$$

where $T$ in $H^{\infty}(2)$ satisfies

$$
T=0 \quad \text { on } \quad Z_{P} \quad \text { the zero set of } P(\cdot, \cdot)
$$

and

$$
T=1 \quad \text { on } \quad Z_{P-1} \quad \text { the pole set of } P(\cdot, \cdot) .
$$

See Section V-A-1 for the proof. Thus, the interpolation problem associated with the classical control problem is as follows.

Find $\mathcal{T} \in \gamma B H^{\infty}(2)$ satisfying the interpolation constraints

$$
\begin{aligned}
& \mathcal{T}(s, w)=\alpha(s) \text { for } \quad(s, w) \in Z_{P} \cup Z_{\beta} \\
& \mathcal{T}(s, w)=\alpha(s)+\beta(s) \text { for }(s, w) \in Z_{P-1} .
\end{aligned}
$$

For convenience set $\mu:=\alpha+\beta$.

The sets $Z_{P}$ and $Z_{P-1}$, being the zero sets of rational functions on $\mathbf{C}^{2}$, typically are one complex dimensional curves in $\mathbf{C}^{2}$ or a finite set of such curves in $\mathbf{C}^{2}$. Thus, we do not have a finite set of interpolating constraints. Agler's Theorem says that for a solution to the $H^{\infty}$ control problem to exist Agler's inequality applied to every finite set of points satisfying this interpolation constraint must have a solution. That is, Agler's inequality is a necessary condition for $H^{\infty}$ control. How close this is to sufficient is not proved. However, it is quite likely that as one takes larger and larger finite sets $F$ of interpolating conditions, as in (5), then the performance bounds $\gamma_{F}$ obtained from Agler's inequality approach being sufficient. What size $F$ is required for a specified accuracy is an issue for computer experiment. 
Next we give more detail, but after that in Section V-B specialize our results to an important case.

1) Proof of (17): The derivation is a trivial extension of the common one variable $H^{\infty}$ control fact (see [11, Lemma 7.2.1]) which in this context says that internal stability is equivalent to: for each $w \in \operatorname{Disk}_{1}$ the functions of $s, w$

$$
T, \quad Q=\frac{P K}{1+P K}, \quad S=T-1, \quad P S=\frac{1}{1+P K} P
$$

are in $H^{\infty}(1)$ with respect to the variable $s$. This by Lemma 4.1 implies $T, Q, S, P S$ as functions of the two variables $s, w$ are in $H^{\infty}(2)$. Now $P(T-1)=P S \varepsilon H^{\infty}(2)$ and $P Q=T$ both being in $H^{\infty}(2)$ are equivalent to the interpolation condition (17).

2) Formulas for $\alpha, \beta$ in equation (16): To implement the method one needs formulas for $\alpha$ and $\beta$ in the representation (16) for $\mathcal{T}$. These formulas are as follows. Select rational $\alpha, \beta, c_{0}$ in $H^{\infty}(1)$ with $c_{0}^{-1} \in$ $H^{\infty}(1)$ which satisfy spectral factorization conditions

$$
\begin{aligned}
\left|c_{0}\right|^{2} & =\left[\tilde{\gamma}^{2}-\frac{\left|\rho_{1}\right|^{4}}{\left|\rho_{1}\right|^{2}+\left|\rho_{2}\right|^{2}}\right] \frac{1}{\gamma^{2}} \\
|\alpha|^{2} & =\frac{\left|\rho_{1}\right|^{4}}{\left|\rho_{1}\right|^{2}+\left|\rho_{2}\right|^{2}} \frac{1}{\left|c_{0}\right|^{2}} \\
|\beta|^{2} & =\left[\left|\rho_{1}\right|^{2}+\left|\rho_{2}\right|^{2}\right] \frac{1}{\left|c_{0}\right|^{2}}
\end{aligned}
$$

$\bar{\alpha} \beta$ is real valued

on the $j \omega$ axis. That $c_{0}^{-1}$ has no poles on the $j \omega$-axis is guaranteed by picking $\tilde{\gamma}$ to satisfy

$$
\tilde{\gamma}^{2}>\sup _{w} \frac{\left|\rho_{1}\right|^{4}}{\left|\rho_{1}\right|^{2}+\left|\rho_{2}\right|^{2}} .
$$

Once this constraint is satisfied one can always find $c_{0}, \alpha, \beta$ meeting all of the conditions. To see this we only need to accommodate (21), since the rest are routine spectral factorizations. If rational $\alpha_{0}, \beta_{0} \in$ $H^{\infty}(1)$ meet conditions (18)-(20), then so do $\psi \alpha_{0}$ and $\phi \alpha_{0}$ for any all pass functions $\psi, \phi \in H^{\infty}$. Choose such $\psi, \phi$ to make

$$
\text { phase } \bar{\psi} \phi=- \text { phase } \bar{\alpha}_{0} \beta_{0},
$$

which is always possible and set $\alpha=\bar{\psi} \alpha_{0}, \quad \beta=\phi \beta_{0}$.

That our formulas for $\alpha, \beta$ do yield the equivalence of (15) and (16) is easy to check by straightforward algebra.

\section{B. Plants With Linear Fractional Uncertainty}

Suppose that the SISO plant $P(s, \delta(s))$ has a linear fractional dependence on $\delta$, namely there are rational functions $a, b, c, d$ meeting the nondegeneracy condition $a(s) b(s)-c(s) d(s) \neq 0$ so that

$$
P(s, w)=\frac{a(s)+b(s) w}{c(s)+d(s) w} .
$$

Suppose the uncertainty $\delta \in d_{0} B H^{\infty}(2)$. Then

$$
\begin{aligned}
Z_{P-1} & =\left\{\left(s, \frac{-c(s)}{d(s)}\right):|s| \leq 1 \text { and }\left|\frac{c(s)}{d(s)}\right| \leq d_{0}\right\} \\
Z_{P} & =\left\{\left(s, \frac{-a(s)}{b(s)}\right):|s| \leq 1 \text { and }\left|\frac{a(s)}{b(s)}\right| \leq d_{0}\right\}
\end{aligned}
$$

and nondegeneracy ensures the two sets do not intersect. Let

$$
v_{\text {pole }}(s):=\frac{-c(s)}{d(s)} \text { and } v_{\text {zero }}(s):=\frac{-a(s)}{d(s)} .
$$

The cleanest way to express the Agler inequality (2) is in terms of $M_{2 \times 2}$ valued functions on $\mathbf{C}^{4}$, and their restriction to a certain subset $\mathcal{R}$ of $\mathbf{C}^{4}$. Define the set $\mathcal{R}$ to be all $(p, r) \in \mathbf{C}^{2}$ satisfying

$$
|p| \leq 1 \text { and }\left|v_{\text {pole }}(p)\right| \leq d_{0}, \quad|r| \leq 1 \text { and }\left|v_{\text {zero }}(r)\right| \leq d_{0} .
$$

\section{Note $\mathcal{R}$ is completely determined by the plant model.}

The unknown is the restriction to $\mathcal{R} \times \mathcal{R}$ of the $M_{2 \times 2}$ valued $\Omega$ defined on $\mathbf{C}^{4}$

$$
\Omega(\{p, r\},\{q, s\}):=\left(\begin{array}{ll}
\Omega^{11}(p, q) & \Omega^{12}(p, s) \\
\overline{\Omega^{12}(q, r)} & \Omega^{22}(r, s)
\end{array}\right) .
$$

It is positive semidefinite in the following sense:

$$
\int_{\mathcal{R} \times \mathcal{R}} \Omega(\{p, r\},\{q, s\}) \overline{f(p, r)} f(q, s) d p d q d r d s \geq 0
$$

for all integrable $f: \mathcal{R} \rightarrow \mathbf{C}^{2}$. Obviously, a necessary condition which is practical to check is: select any finite subset of $\mathcal{R}$ which is not too large, plug it into the formulas above and thereby get a finite-dimensional LMI.

The coefficients in the Agler Inequality are based on the two $M_{2 \times 2}$ valued functions on $\mathbf{C}^{4}$

$S(\{p, r\},\{q, s\})$

$$
:=\left(\begin{array}{cc}
\frac{\gamma^{2}-\overline{\mu(p)} \mu(q)}{1-\bar{p} q} & \frac{\gamma^{2}-\overline{\mu(p)} \alpha(s)}{1-\bar{p} s} \\
\frac{\gamma^{2}-\overline{\alpha(r)} \mu(q)}{1-\bar{r} q} & \frac{\gamma^{2}-\overline{\alpha(r)} \alpha(s)}{1-\bar{r} s}
\end{array}\right)
$$

which is completely determined by the performance specs $\mu$ and $\alpha$ and $P(\{p, r\},\{q, s\})$ defined to be

$$
\left(\begin{array}{cc}
\frac{d_{0}^{2}-\overline{v_{\text {pole }}(p)} v_{\text {pole }}(q)}{1-\bar{p} q} & \frac{d_{0}^{2}-\overline{v_{\text {pole }}(p)} v_{\text {zero }}(s)}{1-\bar{p} s} \\
\frac{d_{0}^{2}-\overline{v_{z e r o}(r)} v_{\text {pole }}(q)}{1-\bar{r} q} & \frac{d_{0}^{2}-\overline{v_{z e r o}(r)} v_{z e r o}(s)}{1-\bar{r} s}
\end{array}\right)
$$

which is completely determined by the plant model. The Agler Inequality $^{2}$ is

$$
0 \leq S-P \circ_{s} \Omega
$$

restricted to $\mathcal{R}$, where recall that ${ }_{S}$ denotes the Schur product (entry by entry product) of two matrices and $\Omega$ must be positive semidefinite in the sense (22). It can be proved using a normal families argument, that the function $\Omega(\{p, r\},\{q, s\})$ may be taken to be anti-analytic in $\{p, r\}$ and analytic in $\{q, s\}$. That is if a nonnegative solution $\Omega$ to (25) exists, then one with these analyticity properties exists.

It might be helpful to note how the classical control problem with no uncertainty fits in formally. This is the case where $d_{0}=0$. In this case the terms involving $\Omega$ drop out, so what remains is $S=\Lambda \geq 0$. Since $d_{0}=0$ the set $\mathcal{R}$ is finite, namely, the plant's pole zero set

$$
\mathcal{R}=\mathcal{R}:=\left\{(p, r) \in \operatorname{Disk}_{2}: P(p)=\infty \text { and } P(r)=0\right\} .
$$

${ }^{2}$ To help one see how this is derived note that the "first form" of Agler's inequality (1) has component

$$
\begin{aligned}
\left(\gamma^{2}-\overline{\mu(p)} \mu(q)\right)=(1-\bar{p} q) & \Lambda^{11}(p, q) \\
& +\left(d_{0}^{2}-\overline{v_{\text {pole }}(p)} v_{\text {pole }}(q)\right) \Omega^{11}(p, q) .
\end{aligned}
$$

due entirely to interpolation constraints at poles. This clearly gives rise to the upper diagonal block of inequality (25). The lower diagonal block corresponds to interpolation constraints at zeros and the off diagonal block accounts for interactions. 
By inspection one sees that $S$ restricted to $\mathcal{R}$ is a Pick matrix; indeed we have the old fashion $H^{\infty}$ control Nevanlinna-Pick solution.

The inequalities (25) suggest an interpretation when $d_{0}>0$. If $\Omega=$ 0 is a solution, then

$$
0 \leq S \quad \text { on } \mathcal{R}
$$

Each choice of $\delta$ gives $\mathcal{R}(s, \delta(s))$ with a finite zero pole set $\mathcal{R}_{F_{\delta}}$ and the statement $0 \leq \mathcal{S}$ on $\mathcal{R}_{F_{\delta}}$ is equivalent to the statement that an $H^{\infty}$ controller meeting the specs exists (for this choice of $\delta$ ). When $d_{0}>0$ many $\delta$ exist, so (27) implies much stronger conditions like $0 \leq \mathcal{S}$ on $\mathcal{R}_{F_{\delta_{1}}} \cup F_{\delta_{2}} \cup F_{\delta_{3}}$, conditions so strong as to be likely unmeetable (a statement which can actually be formalized into a theorem). Fortunately, (25) is more lenient than (27) because $-\mathcal{P} o_{s} \Omega$ can be used to lessen the $0 \leq \mathcal{S}$ constraint.

\section{More General Math THeOREMS}

This article has focused on model matching problems, on the bi-disk. That is because this is one situation for which there is an extremely clean solution. There are other results which have potential and we direct the curious reader to them.

Of direct engineering interest is the case where we do $H^{\infty}$ interpolation on domains in $\mathbf{C}^{1+U}$ of the form $\Omega=\operatorname{Disk}_{1} \times \Delta$, where $\Delta$ is a domain in $\mathbf{C}^{U}$. For example, this occurred in Section III-B with $\Delta$ equal to the ball in $\mathbf{C}^{2}$, denoted $\mathrm{Ball}_{2}$. Fortunately, exactly which such $\Omega$ give problems having an LMI solution of the "nicest possible form" is determined in A. T. Tomerlin's thesis under S. McCullough, cf. [15]. In fact, the case $\Omega=D i s k_{1} \times$ Ball $_{2}$, has a very nice LMI solution.

An example of a Domain which fails the "niceness test" is $\Delta=$ $D_{i s k_{2}}$ the bi-disk, then $\Omega=D i s k_{1} \times D i s k_{2}=: D i s k_{3}$, the tri-disk. However, J. Agler's [1] results on the bi-disk generalize to any polydisk by formulating the interpolation problem with exactly the same constraints (5) as before, but with a different norm. Agler's norm Norm ${ }_{n}$ on $H^{\infty}$ of the Diskn is stronger than the supremum norm, and is not classical (unless $n=1$ or 2 in which case it is the supremum norm). However, Norm $_{n}$ has the property that the solution to the associated interpolation problem reduces to an LMI of dimension comparable to the number of interpolation constraints, and so is potentially solvable. The potential value of this to control theorists is that we have solvable problems and an engineer who studies Norm $_{n}$ might find it approximates something physical. Also of possible interest is work by Alpay, J. Ball, V. Bolotnikov, and T. Trent (see [4], [2]) which puts Agler's results and other SCV results in statespace coordinates.

Secondly, results of Cotlar and Sadosky [5], [6] on interpolation and approximation problems on the polydisk use a performance measure called the bounded mean oscillation (BMO) norm. For a survey see [13]. They show that various BMO norms give elegant results. Their formulas are quite possibly computable, so the main issue is whether or not BMO norms are close to something physical.

\section{ACKNOWLEDGMENT}

The author is most grateful to M. Putinar for assistance with technical issues in SCV connected with the general position assumption and to J. Ball for help with several mathematical points. Thanks are certainly due to C. Sadosky for constantly urging me to find an application for this realm of SCV hotly pursued these days by operator theorists. The author is also grateful to A. Packard and H. Ito for their explanations of LPV control and LFT based control. Also, thanks to $\mathrm{H}$. Kimura for pointing out typos in the original manuscript and for discussions. Thanks are due to B. Bitmead for suggesting the example in Section III-B.

\section{REFERENCES}

[1] J. Agler, "Some interpolation theorems of Nevanlinna-Pick type," Tech. Rep. Univ. California, San Diego, 1980.

[2] D. Alpay, J. Ball, and V. Boltnikov, "On the bi-tangential interpolation problem for contractive valued functions in the polydisk,", to be published.

[3] J. Agler and J. McCarthy. (1997) Nevanlinna-Pick interpolation on the bi-disk. [Online]. Available: http://www.math.wustl.edu/mccarthy/.

[4] J. Ball and T. Trent, "Unitary colligations reproducing kernel Hilbert spaces and Nevannlina Pick interpolation in several variables," J. Functional Anal., to be published.

[5] M. Cotlar and C. Sadosky, "Nehari and Nevanlinna-Pick problems and homomorphic extensions in the polydisk in terms of restricted BMO," J. Functional Anal., vol. 124, pp. 205-210, 1994.

[6] _ - "Two distinguished subspaces of product BMO and the Nehari-AAK theory for Hankel operators in the torus," Integral Eqs. Operator Theory, vol. 26, pp. 273-304, 1996.

[7] B. Cole and J. Wermer, "Pick interpolation, von Neumann inequalities, and hyperconvex sets,", 1996, to be published.

[8] B. A. Francis, A Course in $H^{\infty}$ Control, ser. Lecture Notes in Control and Info Sci.. New York: Springer-Verlag, 1987, vol. 88, p. 156.

[9] J. W. Helton, Operator Theory, Analytic Functions, Matrices and Electrical Engineering, ser. CBMS Regional Conference Series in Mathematics, No. 68. Providence, RI: American Math Society, 1987.

[10] J. W. Helton, "A type of gain scheduling which converts to a "Classical' problem in several complex variables," in Proc. Amer. Control Conf. 1999, San Diego, CA.

[11] J. W. Helton and O. Merino, "Classical control using $H^{\infty}$ methods," SIAM, p. 292, 1998.

[12] S. G. Krantz, Function Theory of Several Complex Variables. New York: Wiley, 1982.

[13] C. Sadosky, Liftings of Kernels Shift-Invariant in Scattering Systems in Holomorphic Spaces. Cambridge, U.K.: Cambridge Univ. Press, 1998, MSRI Pub. 33, pp. 302-306. A paper that directs one to other readings.

[14] A. Sideris and M. G. Safonov, "Robust synthesis of SISO feedback control systems using conformal maps and $H_{\infty}$ theory-A design example," in Proc. Amer. Control Conf., Seattle, WA, June 1986.

[15] A. T. Tomerlin, "Products of Nevanlinna-Pick kernals and operator colligations," IEOT, to be published. 